\title{
Modelo predictivo para mortalidad en adultos hospitalizados con neumonía adquirida en la comunidad
}

\author{
Predictive model for mortality in adults hospitalized for community-acquired pneumonia
}

\author{
Renata Báez-Saldaña ${ }^{1,2 *}$, Carlos Gómez-Zamora ${ }^{1,2}$, Leticia Ferreyra-Reyes ${ }^{3}$, Norma Mongua-Rodríguez ${ }^{3}$ \\ y Lourdes García- García ${ }^{3}$ \\ ${ }^{1}$ Servicio Clínico de Neumología Oncológica, Instituto Nacional de Enfermedades Respiratorias, Ciudad de México; ${ }^{2}$ División de Posgrado, Facultad \\ de Medicina, Universidad Nacional Autónoma de México, Ciudad de México; ${ }^{3}$ Instituto Nacional de Salud Pública, Cuernavaca, Morelos. México
}

\begin{abstract}
Resumen
Antecedentes: Existe escasa información sobre variables predictoras para mortalidad por neumonía en población mexicana. Objetivo: Identificar un modelo de variables predictoras para mortalidad en adultos hospitalizados por neumonía adquirida en la comunidad (NAC). Método: Estudio de casos y controles de pacientes hospitalizados por NAC. Los casos fueron pacientes con NAC que fallecieron durante la hospitalización y los controles fueron pacientes que no fallecieron. Los pacientes se siguieron durante el tiempo de estudio hasta el egreso o el desenlace de cada uno. La asociación entre las variables independientes (clínicas, índices de gravedad para neumonía) con la variable desenlace (muerte hospitalaria) se evaluó mediante regresión logística. Resultados: Las variables asociadas a mortalidad fueron la edad estratificada de 50-70 años (odds ratio [OR]: 2.35; intervalo de confianza del 95\% [IC 95\%]: 1.06-5.17) y > 70 años (OR: 2.75; IC 95\%: 1.18-6.37), ser mujer (OR: 2.05; IC 95\%: 1.11-3.8), antecedente de hipertensión arterial (OR: 0.40; IC 95\%: 0.20-0.80), taquicardia (OR: 2.68; IC 95\%: 1.16-6.17), taquipnea (OR: 2.85; IC 95\%: 1.54-5.29), leucocitos $\geq 12,000$ (OR: 2.17; IC 95\%: 1.21-3.87) y nitrógeno de la urea $>30 \mathrm{mg} / \mathrm{dl}$ (OR: 4.85; IC 95\%: 2.55-9.24). Conclusiones: El modelo de riesgo que se propone es fácil de documentar con la historia clínica y las pruebas de laboratorio habituales.
\end{abstract}

Palabras clave: Adultos. Factores de riesgo. Mortalidad. Neumonía adquirida en la comunidad.

\begin{abstract}
Introduction: There is lack of information about predictive variables for pneumonia mortality in the Mexican population. Objective: To identify a model for predictive variables for mortality in adults hospitalized with community acquired pneumonia (CAP). Material and methods: Case-control study of patients hospitalized with CAP. The cases were incident cases with CAP that died during hospitalization and controls were those that did not die. The patients were followed during the study time until the discharge or outcome of each patient. The association between the independent variables (clinical, severity indices for pneumonia) with the outcome variable (in-hospital mortality) was evaluated by logistic regression. Results: The model of associated variables with mortality were: stratified age from 50-70 years (odds ratio [OR]: 2.35; 95\% confidence interval [95\% Cl: 1.06-5.17) and age $>70$ years (OR: 2.75; 95\% Cl: 1.18-6.37), woman (OR: 2.05; 95\% Cl: 1.11-3.8), antecedent of arterial hypertension (OR: 0.40; 95\% Cl: 0.20-0.80), tachycardia (OR: 2.68; 95\% Cl: 1.16-6.17), tachypnea (OR: 2.85; 95\% Cl: 1.54-5.29),
\end{abstract}

\footnotetext{
Correspondencia:

*Renata Báez-Saldaña

Tlalpan, 4502

Col. Sección XVI

Fecha de recepción: 27-07-2019

Cir Cir. 2020;88(5):584-590

C.P. 14080 , Ciudad de México, México

Fecha de aceptación: 18-05-2020

Contents available at PubMed

E-mail: baezrd@unam.mx

DOI: $10.24875 / C I R U .20001451$

www.cirugiaycirujanos.com

0009-7411/@ 2020 Academia Mexicana de Cirugía. Publicado por Permanyer. Este es un artículo open access bajo la licencia CC BY-NC-ND (http://creativecommons.org/licenses/by-nc-nd/4.0/).
} 
leukocytes $\geq 12,000$ (OR: 2.17; 95\% Cl: 1.21-3.87) and urea nitrogen > $30 \mathrm{mg} / \mathrm{dL}$ (OR: 4.85; 95\% Cl: 2.55-9.24). Conclusions: The proposed risk model is easy to document with clinical history and routine clinical laboratory variables.

Key words: Adults. Risk factors. Mortality. Community acquired pneumonia.

\section{Introducción}

La neumonía adquirida en la comunidad (NAC) es una enfermedad frecuente y potencialmente grave. Aunque la tasa de mortalidad por neumonía ha disminuido desde la introducción del tratamiento antibiótico en la década de 1940, aún se ubica como una de las principales causas de morbilidad y mortalidad en todo el mundo.

En México, en el año 2014 ocupó el lugar 19 como causa de morbilidad, con 174,748 casos y una tasa de 1.5 por 100,000 habitantes; así mismo, en el año 2013 fue la causa número 12 de mortalidad, con 17,417 casos, lo que correspondió al $2.8 \%$ de todas las causas de mortalidad en el país ${ }^{1}$. En el Instituto Nacional de Enfermedades Respiratorias (INER) es la segunda causa de atención en la unidad de urgencias y la primera de morbilidad hospitalaria (datos obtenidos de la Dirección Médica del INER, 2013-2017).

La evaluación de la gravedad es fundamental, ya que la identificación temprana de los individuos con riesgo elevado de muerte puede ayudar a decidir el sitio de la atención médica y el esquema del tratamiento. Por ello, las guías clínicas para la NAC inician el algoritmo de tratamiento con la evaluación de la gravedad mediante reglas de predicción para la estratificación del riesgo de los pacientes ${ }^{2-4}$. Los modelos de predicción del riesgo mejor validados y que se utilizan con más frecuencia son la escala CURB- $65^{5}$ (confusión, nitrógeno ureico $\geq 20 \mathrm{mg} / \mathrm{dl}$, frecuencia respiratoria $\geq 30$ respiraciones por minuto [r.p.m.], presión sistólica $<90 \mathrm{mmHg}$ o diastólica $\leq 60 \mathrm{mmHg}$, y edad mayor de 65 años) y el índice de gravedad para neumonía (PSI, Pneumonia Severity Index) ${ }^{6}$. Estos modelos de predicción estadística fueron elaborados a partir de la identificación de variables asociadas a mortalidad y permiten identificar a los pacientes con riesgo bajo que pueden tratarse como ambulatorios y con ello disminuir los costos de atención. Estudios previos han demostrado su eficacia y seguridad para reducir la hospitalización en los pacientes de riesgo bajo para mortalidad; sin embargo, la proporción de pacientes hospitalizados clasificados en este estrato se ubica entre el $31 \%$ y el $62.5 \%$.
En pacientes mexicanos, los factores de riesgo asociados a mortalidad por neumonía han sido poco explorados y su reconocimiento es fundamental para el tratamiento apropiado de acuerdo con el caso, así como para la decisión de hospitalización. En este contexto, el objetivo del presente estudio fue identificar y evaluar un modelo de variables predictoras para mortalidad en pacientes adultos hospitalizados con NAC y medir la proporción de los clasificados como de riesgo bajo para mortalidad conforme a los índices PSI y CURB-65.

\section{Método}

El protocolo fue aprobado por el Comité de Ética en Investigación institucional. Los resultados se manejaron de tal forma que garantizaron la protección de los derechos individuales y la confidencialidad.

\section{Diseño, pacientes, lugar y período de estudio}

Estudio de casos y controles de pacientes hospitalizados consecutivos con diagnóstico de NAC, de 2006 a 2013, en un hospital de referencia de enfermedades respiratorias en la Ciudad de México.

Se incluyeron casos hospitalizados con diagnóstico de NAC, definida como enfermedad aguda en la que el paciente presenta una opacidad pulmonar nueva asociada al menos a uno de los siguientes signos o síntomas: tos de reciente inicio, fiebre o hipotermia, leucocitosis, o leucocitopenia, desviación a la izquierda, para los que no hay otra explicación y la enfermedad es la causa principal de la admisión hospitalaria y se tratará como neumonía9. Se excluyeron los pacientes con neumonía posobstructiva, antecedente de hospitalización en las 2 semanas previas a la admisión, neumonía nosocomial, neumonía por influenza y cualquier tipo de inmunosupresión (ya sea congénita o adquirida) o bajo cualquier tratamiento inmunosupresor.

\section{Definición de caso}

Paciente con diagnóstico de ingreso de NAC que falleció durante su hospitalización. 


\section{Definición de control}

Paciente hospitalizado con diagnóstico de ingreso de NAC que egresó por curación.

Los pacientes se incluyeron consecutivamente conforme cumplieron con los criterios de inclusión. La decisión del sitio de tratamiento de los pacientes fue a criterio del médico tratante del servicio de urgencias, sin influencia de la implementación de las reglas de predicción para mortalidad.

\section{Tamaño de muestra y poder}

Se estableció un tamaño de muestra mínimo necesario para identificar una odds ratio (OR) diferente de 1 , un error alfa de 0.05 , una potencia de 0.90 , cuatro controles por caso y considerando la prueba de ji al cuadrado sin corrección de continuidad. Así, fueron necesarios 38 casos y 152 controles $^{10}$.

Se revisó cada caso a fin de que se cumplieran los criterios de diagnóstico de NAC y los datos se recolectaron en una hoja de trabajo estandarizada para el estudio. Los pacientes se siguieron durante el tiempo de estudio hasta el egreso o el desenlace de cada paciente.

La variable «desenlace» fue la muerte hospitalaria. Las variables independientes fueron variables sociodemográficas, características clínicas (comorbilidad, síntomas, tiempo de padecimiento, tiempo entre el inicio del padecimiento y el inicio del tratamiento), antecedentes de vacunación para influenza y neumococo, derrame pleural, obesidad (índice de masa corporal [IMC] $>30 \mathrm{~kg} / \mathrm{m}^{2}$ ) y desnutrición (IMC $<18.5 \mathrm{~kg} / \mathrm{m}^{2}$ para mujeresy $<20 \mathrm{~kg} / \mathrm{m}^{2}$ para hombres), pruebas de laboratorio clínico habituales para estos pacientes, tratamiento antibiótico inicial, microorganismos aislados y calificación de los índices de gravedad PSI ${ }^{6}$ y CURB65 ${ }^{5}$. Para calcular el PSI se van sumando puntos según la edad, el sexo, la presencia de comorbilidad y las alteraciones presentes en la exploración física y en los estudios de laboratorio y radiológicos. Con el puntaje obtenido, los pacientes son estratificados en categorías de I a V según su gravedad. La clase I corresponde a pacientes menores de 50 años sin enfermedad neoplásica, hepática, insuficiencia cardiaca congestiva ni enfermedad cerebrovascular o renal, con signos vitales normales o discretamente alterados, y sin trastorno del estado mental. Las clases Il a V seasignan de acuerdo con tres variables demográficas (edad, sexo y residencia en asilo), cinco condiciones comórbidas, cinco hallazgos en la exploración física y siete hallazgos de laboratorio o radiográficos. La clase de riesgo I corresponde a 0 puntos, la clase II entre 1 y 70 puntos, la clase III entre 71 y 90 puntos, la clase IV entre 91 y 130 puntos y la clase $V$ más de 130 puntos.

La escala CURB-65 se basa en la evaluación de cuatro variables pronósticas esenciales, además de edad mayor de 65 años: confusión, definida como una calificación de 8 o menos en la prueba del estado mental abreviado; nitrógeno ureico $\geq 20 \mathrm{mg} / \mathrm{dl}$, frecuencia respiratoria $\geq 30$ r.p.m. y presión sistólica $<90 \mathrm{mmHg}$ o diastólica $\leq 60 \mathrm{mmHg}$.

Cada variable presente representa 1 punto, y los pacientes con dos o más de estos factores pronósticos tienen un riesgo muy elevado de muerte y deben ser referidos de inmediato al hospital, ya que ello decide el ingreso a la unidad de cuidados intensivos.

El tratamiento antimicrobiano inicial en todos los casos se apegóa las guías internacionales y nacionales de práctica clínica para la NAC. Los esquemas que se utilizaron más frecuentemente fueron cefalosporina de tercera generación más macrólido o fluoroquinolona respiratoria, ambos inicialmente porvía intravenosa. La decisión de un esquema u otro fue a criterio del médico tratante y de la disponibilidad de antimicrobianos en la institución.

\section{Análisis estadístico}

Se usó el paquete estadístico STATA 15.1.0 (College Station, Texas, USA). Para resumir los datos se utilizó la estadística descriptiva apropiada a cada variable. Las variables continuas se expresaron como mediana (intervalo intercuartil) y las variables categóricas como frecuencias y porcentajes.

La asociación de las diferentes variables independientes (clínicas y de laboratorio) con la variable desenlace (muerte hospitalaria) se evaluó mediante regresión logística conforme a la metodología de Hosmer-Lemeshow. Inicialmente se realizó un análisis bivariado, a partir del cual se seleccionaron variables según su significancia estadística $(p \leq 0.2)$ y su plausibilidad biológica, mismas que se incluyeron en un modelo para análisis multivariado. Las razones de momios se expresaron con un intervalo de confianza del 95\% (IC 95\%). Para evaluar el modelo se realizó la prueba de bondad de ajuste, y se determinaron la sensibilidad, la especificidad y los valores predictivos del modelo, así como el área bajo la curva.

\section{Resultados}

Se incluyeron pacientes con NAC: 87 casos con defunción hospitalaria y 230 controles vivos al egreso. 
Tabla 1. Características basales de la población de estudio con respecto al estado vital

\begin{tabular}{|c|c|c|c|c|c|}
\hline Características clínicas & $\begin{array}{l}\text { Población total } \\
(\mathrm{n}=317) \mathrm{n}(\%)\end{array}$ & $\begin{array}{c}\text { Casos }(n=87) \\
n(\%)\end{array}$ & $\begin{array}{c}\text { Controles } \\
(n=230) n(\%)\end{array}$ & OR (IC 95\%) & $\mathrm{p}$ \\
\hline \multicolumn{6}{|l|}{ Generales } \\
\hline Edad en años, mediana (intervalo intercuartil 25-75) & $58(41-73)$ & $64(47-79)$ & $55(38-71)$ & $1.0(1.0-1.03)$ & 0.006 \\
\hline Edad > 50 años & $196(61.8)$ & $63(72.4)$ & $133(57.8)$ & $1.91(1.12-3.28)$ & 0.018 \\
\hline \multicolumn{6}{|l|}{ Sexo } \\
\hline Mujer & $144(45.4)$ & $45(51.7)$ & $99(43)$ & $1.41(0.86-2.32)$ & 0.167 \\
\hline Hombre & $173(54.6)$ & $42(48.3)$ & $131(57)$ & & \\
\hline \multicolumn{6}{|l|}{ Comorbilidad } \\
\hline Al menos una comorbilidad & $189(59.6)$ & $54(62)$ & $135(58.7)$ & $1.15(0.69-1.91)$ & 0.585 \\
\hline Diabetes & $87(27.4)$ & $19(21.8)$ & $68(29.6)$ & $0.67(0.37-1.19)$ & 0.171 \\
\hline Hipertensión arterial & $96(30.4)$ & $9(10.3)$ & $36(15.7)$ & $0.67(0.38-1.17)$ & 0.160 \\
\hline Cardiovascular & $33(10.4)$ & $13(15)$ & $20(8.7)$ & $1.84(0.87-3.89)$ & 0.108 \\
\hline Cerebrovascular & $43(13.6)$ & $18(20.7)$ & $25(10.9)$ & $2.13(1.10-4.16)$ & 0.025 \\
\hline Neoplásica & $11(3.5)$ & $2(2.3)$ & $9(3.9)$ & $0.58(0.12-2.73)$ & 0.489 \\
\hline Tabaquismo actual o pasado & $145(45.7)$ & $40(46)$ & $105(45.7)$ & $1.01(0.62-1.66)$ & 0.959 \\
\hline Exposición a humo de leña & $87(27.4)$ & $28(32.2)$ & $59(25.7)$ & $1.38(0.80-2.36)$ & 0.246 \\
\hline Antibiótico previo & $125(39.4)$ & $38(43.7)$ & $87(37.8)$ & $1.27(0.78-2-10)$ & 0.342 \\
\hline
\end{tabular}

Los casos tuvieron mayor edad que los vivos al egreso, con una mediana de 64 vs. 55 años, respectivamente $(p<0.05)$. La edad como variable continua y estratificada se asoció significativamente con la mortalidad. A partir de los 50 años, el riesgo de mortalidad se incrementa de forma proporcional conforme aumenta la edad: en el estrato de 50-70 años la OR fue de 1.66 (IC 95\%: 0.89-3) y en los mayores de 70 años la OR fue de 2.22 (IC 95\%: 1.20-4.12). Entre los casos hubo más mujeres que hombres, sin diferencia significativa. En la población total, tener al menos una comorbilidad (diabetes, hipertensión arterial sistémica, enfermedad cerebrovascular, etc.) estuvo presente en 189 (59.6\%) pacientes, sin diferencias significativas entre los casos y los controles. Tener enfermedad cerebrovascular se asoció a mortalidad (OR: 2.13; IC 95\%: 1.10-4.16). No hubo diferencias entre los casos y los controles con respecto a los antecedentes de exposición al humo de tabaco o de leña (Tabla 1).

El análisis crudo de la asociación entre las alteraciones en los signos clínicos y las variables de laboratorio con la mortalidad demostró que muchas se asociaron positivamente, como la frecuencia cardiaca $>124$ latidos por minuto (l.p.m.), la frecuencia respiratoria $>30$ r.p.m., los leucocitos totales $>12,000 \mathrm{~mm}^{3}$, el nitrógeno de la urea $\geq 30 \mathrm{mg} / \mathrm{dl}$ y el $\mathrm{pH}$ arterial $<7.35$, entre otros. Se identificó el patógeno respiratorio en el $14.2 \%$ de la población total, sin diferencias significativas entre los dos grupos de estudio. La clase $\mathrm{PSI}>3$ y un puntaje CURB-65 $>1$ se asociaron fuertemente a mortalidad (Tabla 2).
El modelo multivariado para mortalidad demostró que la edad estratificada sigue fuertemente asociada, así como otras variables como ser mujer, frecuencia cardiaca > 124 I.p.m., frecuencia respiratoria $>30$ r.p.m., leucocitos totales $\geq 12,000$ y nitrógeno de la urea $>30 \mathrm{mg} / \mathrm{dl}$. Tener el antecedente de hipertensión arterial sistémica persiste como variable protectora para mortalidad en este modelo (Tabla 3). Respecto a la evaluación global del modelo, este cumplió con el criterio de bondad de ajuste ( $p=0.7417)$ y presentó una sensibilidad del $38.37 \%$, una especificidad del $93.48 \%$, un valor predictivo positivo del $68.75 \%$ y un valor predictivo negativo del $80.22 \%$. El valor predictivo del modelo evaluado mediante el área bajo la curva fue de 0.78 .

En la distribución de los riesgos de mortalidad para los índices PSI y CURB-65 en los casos de defunción destaca una tendencia directamente proporcional a la frecuencia de mortalidad observada conforme se incrementa cada nivel de riesgo. Así mismo, documentamos que, del total de la muestra estudiada, no cumplieron los criterios de hospitalización conforme al PSI clase I y II 113 (35.6\%) pacientes, y para un CURB-65 de 0 y 1 fueron 156 (49.2\%) (Tabla 4).

\section{Discusión}

El modelo que se propone como predictor de mortalidad para NAC incluye siete variables que son fáciles de documentar: cinco de ellas se obtienen fácilmente de la historia clínica y la toma de signos 
Tabla 2. Signos vitales y variables de laboratorio clínico seleccionadas con respecto al estado vital de la población de estudio

\begin{tabular}{|c|c|c|c|c|}
\hline & Casos $(n=87) n(\%)$ & Controles $(n=230) n(\%)$ & OR (IC 95\%) & $p$ \\
\hline \multicolumn{5}{|l|}{ Signos clínicos } \\
\hline Presión sistólica < 90 mmHg & $13(14.9)$ & $17(7.4)$ & $2.13(0.99-4.60)$ & 0.054 \\
\hline Frecuencia cardiaca > 124 I.p.m. & $19(21.8)$ & $24(10.4)$ & $2.39(1.24-4.65)$ & 0.010 \\
\hline Frecuencia respiratoria $\geq 30$ r.p.m. & $38(46.7)$ & $48(20.9)$ & $2.86(1.68-4.86)$ & 0.001 \\
\hline Derrame pleural & $5(5.8)$ & $37(16.1)$ & $0.32(0.12-0.84$ & 0.020 \\
\hline \multicolumn{5}{|l|}{ Laboratorio clínico } \\
\hline Leucocitos totales $\geq 12,000 / \mathrm{mm}^{3}$ & $56(64.4)$ & $106(46.1)$ & $2.11(1.3-3.5)$ & 0.004 \\
\hline Leucocitos totales $\leq 3,500$ & $4(4.6)$ & $4(1.7)$ & $2.76(0.68-11.2)$ & 0.155 \\
\hline Neutrófilos totales $\geq 9,500$ & $52(59.8)$ & $107(46.5)$ & $1.70(1.03-2.82)$ & 0.036 \\
\hline Hematocrito $<30 \%$ & $14(16.1)$ & $16(7)$ & $2.6(1.2-5.5)$ & 0.016 \\
\hline Glucosa > 250 mg/dl & $8(9.2)$ & $17(7.4)$ & $1.3(0.5-3)$ & 0.596 \\
\hline Nitrógeno en urea $\geq 30$ mg/dl & $42(48.3)$ & $36(15.7)$ & $5(2.9-8.7)$ & 0.001 \\
\hline Sodio $<130 \mathrm{mmol} / \mathrm{l}$ & $6(7)$ & $21(9)$ & $0.74(0.29-1.89)$ & 0.526 \\
\hline Albúmina sérica $<3.0 \mathrm{~g} / \mathrm{dl} \mathrm{N} / \mathrm{n}$ & $66 / 85(77.7)$ & $153 / 289(73.2)$ & $1.27(0.70-2.3)$ & 0.429 \\
\hline $\mathrm{pH}$ arterial $<7.35$ & $14(16.1)$ & $17(7.4)$ & $2.40(1.13-5.12)$ & 0.023 \\
\hline $\mathrm{SpO}_{2}<88 \%$ & $63(72.4)$ & $116(50.4)$ & $2.57(1.51-4.41)$ & 0.001 \\
\hline Identificación microbiológica & $14(16.09)$ & $31(13.5)$ & $1.23(0.62-2.45)$ & 0.552 \\
\hline PSI clase III & $62(70.1)$ & $75(32.6)$ & $4.85(2.84-8.28)$ & 0.000 \\
\hline CURB-65 > 1 & $65(74.7)$ & $96(41.7)$ & $4.12(2.38-7.15)$ & 0.000 \\
\hline
\end{tabular}

IC 95\%: intervalo de confianza del 95\%; OR: odds ratio; $\mathrm{SpO}_{2}$ : saturación de oxígeno en sangre.

Tabla 3. Análisis multivariado para mortalidad por neumonía adquirida en la comunidad

\begin{tabular}{lcc}
\hline Características clínicas & OR (IC 95\%) & p \\
\hline Edad estratificada & & \\
$<50$ años & Referencia & ] 0.034 \\
$50-70$ años & $2.35(1.06-5.17)$ & 0.019 \\
$>70$ años & $2.75(1.18-6.37)$ & \\
& & \\
Mujer & $2.05(1.11-3.8)$ & 0.021 \\
Hipertensión arterial & $0.40(0.20-0.80)$ & 0.010 \\
Frecuencia cardiaca > 124 I.p.m. & $2.68(1.16-6.17)$ & 0.021 \\
Frecuencia respiratoria > 30 r.p.m. & $2.85(1.54-5.29)$ & 0.001 \\
Leucocitos totales $\geq 12,000$ & $2.17(1.21-3.87)$ & 0.009 \\
Nitrógeno en urea > 30 mg/dl & $4.85(2.55-9.24)$ & 0.000 \\
\hline
\end{tabular}

vitales, y las otras dos son variables de laboratorio clínico disponibles en cualquier unidad de atención médica. Demostró ser un buen modelo según los criterios que se estudiaron para su evaluación, como la prueba de bondad de ajuste y el área bajo la curva.

En el análisis bivariado, muchas de las variables que resultaron asociadas a mortalidad para NAC son las ya descritas en otras poblaciones y las incluidas en los índices de predicción de mortalidad $\mathrm{PSI}^{6}$ y CURB-655, como comorbilidad neurológica y cardiovascular, signos clínicos como frecuencia cardiaca $>124$ I.p.m., frecuencia respiratoria > 30 r.p.m. y presión arterial sistólica $<90 \mathrm{mmHg}$, así como variables
Tabla 4. Distribución de riesgos y mortalidad observada para PSI y CURB-65 en la población total y en las defunciones

\begin{tabular}{lcc}
\hline Gravedad & $\begin{array}{c}\text { Población total }(\mathbf{n}=\mathbf{3 1 7}) \\
\mathbf{n}(\%)\end{array}$ & $\begin{array}{c}\text { Defunciones }(\mathbf{n}=\mathbf{8 7}) \\
\mathbf{n}(\%)\end{array}$ \\
\hline PSI clase I & $52(16.4)$ & $3(5.8)$ \\
PSI clase II & $61(19.2)$ & $7(11.5)$ \\
PSI clase III & $68(21.5)$ & $16(23.5)$ \\
PSI clase IV & $94(29.6)$ & $39(41.5)$ \\
PSI clase V & $42(13.3)$ & $22(52.4)$ \\
CURB 0 & $80(25.2)$ & $10(12.5)$ \\
CURB 1 & $76(24)$ & $12(15.8)$ \\
CURB 2 & $88(27.8)$ & $31(35.2)$ \\
CURB 3 & $55(17.4)$ & $23(41.8)$ \\
CURB 4 & $16(5)$ & $9(56.3)$ \\
CURB 5 & $2(0.63)$ & $2(100)$ \\
\hline
\end{tabular}

de laboratorio como nitrógeno de la urea $>30 \mathrm{mg} / \mathrm{dl}$ $y$ hematocrito $<30 \%{ }^{11}$. Otras variables no incluidas en dichos índices de predicción, como leucocitos totales $>12,000$ y neutrófilos totales $>9,500$, también salieron asociadas en nuestra población. La albúmina no se asoció a mortalidad en nuestro estudio y el derrame pleural resultó ser un factor protector (OR: 0.32; IC 95\%: 0.12-0.84) para mortalidad por NAC. En 
contraste, en el estudio de validación de la regla de predicción CURB-65, la albúmina y el derrame pleural resultaron asociados a mortalidad en el análisis bivariado, aunque fueron muy pocos sujetos evaluados para esas variables 5 . Adicionalmente, un PSI de clase $>$ III y un CURB-65 > 1 punto también son útiles debido a que ambos se vieron fuertemente asociados a mortalidad, con unas OR de 4.85 (IC 95\%: 2.84-8.28) y 4.12 (IC 95\%: 2.38-7.15), respectivamente, lo que sugiere que el perfil de riesgo de nuestra población, en términos generales, no es diferente del escenario del cual derivaron dichas reglas de predicción, lo que impacta positivamente sobre su validez.

En el modelo multivariado se sigue confirmando la observación de que la edad mayor de 50 años es un fuerte predictor de mortalidad, junto con otras variables como ser mujer y tener unos leucocitos totales $\geq 12,000$. El antecedente de hipertensión arterial sistémica se comporta como variable protectora para mortalidad en este modelo. Respecto a esta última variable, la única explicación que tenemos es que se puede deber al efecto opuesto que tiene la hipotensión como factor de riesgo, y consideramos que es biológicamente plausible. Lo anterior, hasta donde sabemos, es algo no descrito que merece ser explorado, pues persiste cuando se ajusta por otras variables como la edad, y precisamente en los mayores de 50 años la prevalencia del antecedente de hipertensión arterial es mucho mayor que en los menores de 50 años (42.6 vs. $10.7 \%)$.

Con la finalidad de mejorar el desenlace de los pacientes que sufren NAC, a partir de 1993 algunas sociedades médicas de los Estados Unidos de América y de otras partes del mundo elaboraron diferentes guías clínicas para la atención de estos pacientes ${ }^{2-4}$, en las que se proponen recomendaciones para el diagnóstico, el tratamiento y la prevención de la NAC, cuyo cumplimiento ha evidenciado un impacto positivo sobre el desenlace. Sin embargo, aún existe el problema de la decisión de qué pacientes hospitalizar y cuáles pueden tratarse de forma ambulatoria, lo que indiscutiblemente impacta sobre los costos de atención.

Los índices de predicción pronóstica, como PSI y CURB-65, aunque están validados para mortalidad, se utilizan principalmente para identificar los casos de menor riesgo y que pueden tratarse de forma ambulatoria sin incrementar el riesgo de muerte. Para el PSI, se indica que los pacientes de las clases I y II generalmente no requieren hospitalización, aquellos en clase III pueden requerir hospitalización muy breve, y los de las clases IV y $V$ generalmente requieren ser hospitalizados. Para el CURB-65, se recomienda hospitalizar a los que tienen 2 puntos o más. Sin embargo, es muy frecuente la hospitalización de pacientes clasificados como de riesgo bajo por diversas razones. En nuestro estudio, el $35.6 \%$ y el $49.2 \%$ de los casos no tuvieron criterios de hospitalización de acuerdo con PSI y CURB-65, respectivamente, proporción que se ubica entre las cifras descritas por otros autores. En el estudio internacional CAPO de calidad de la atención médica, el $26 \%$ de los casos no cumplieron el criterio de hospitalización de acuerdo con el PSI ${ }^{12}$, y otros estudios ubican este mismo indicador entre el $31 \%$ y el $62.5 \%$.7.8. Las razones para la hospitalización en la mayoría de nuestros pacientes que conforme a Ios criterios PSI y CURB-65 no la requirieron fueron hipoxemia, necesidad de tratar alguna comorbilidad, derrame pleural paraneumónico, afección radiológica multilobular bilateral o intolerancia oral. Sin embargo, es probable que en muchos casos se haya estimado una mayor necesidad de hospitalización. Al respecto, hay evidencia de que aproximadamente en el $20 \%$ de los casos hospitalizados con riesgo bajo para mortalidad no se documenta ninguna razón para ello, excepto la decisión del médico tratante ${ }^{13}$. Este es un aspecto que hay que evaluar en nuestra institución dadas sus características, y aunque el PSI es un buen predictor de riesgo de mortalidad al momento de la hospitalización, puede no ser útil en algunos casos, pues no toma en cuenta factores de tipo social ni la necesidad de tratar otra comorbilidad (Tabla 4).

Las limitaciones del presente estudio son su diseño de casos y controles y el tamaño de muestra, comparado con las grandes cohortes de las que derivaron los índices de predicción; además, no hubo seguimiento al egreso y los resultados no aplican a pacientes ambulatorios. No pudimos evaluar la contribución del antecedente de vacunación contra el neumococo por falta de información al respecto.

\section{Conclusiones}

En el modelo de riesgo que se propone, la edad superior a 50 años, ser mujer, la frecuencia cardiaca $>124$ I.p.m., la frecuencia respiratoria > 30 r.p.m., los leucocitos $>12,000$ y el nitrógeno de la urea $>20 \mathrm{mg} / \mathrm{dl}$ se asociaron positivamente con la mortalidad. El antecedente de hipertensión arterial sistémica fue protector para la mortalidad. Las variables del modelo son fáciles de documentar con la historia clínica y las 
pruebas de laboratorio habituales en los pacientes hospitalizados con NAC.

Destaca una elevada frecuencia de pacientes hospitalizados con criterios de riesgo bajo según PSI y CURB-65: el $35.6 \%$ y el $49.2 \%$, respectivamente

\section{Financiamiento}

Los autores no recibieron patrocinio para el desarrollo de este manuscrito.

\section{Conflicto de intereses}

Los autores declaran no tener conflicto de intereses.

\section{Responsabilidades éticas}

Protección de personas y animales. Los autores declaran que para esta investigación no se han realizado experimentos en seres humanos ni en animales.

Confidencialidad de los datos. Los autores declaran que han seguido los protocolos de su centro de trabajo sobre la publicación de datos de pacientes.

Derecho a la privacidad y consentimiento informado. Los autores han obtenido el consentimiento informado de los pacientes y/o sujetos referidos en el artículo. Este documento obra en poder del autor de correspondencia.

\section{Bibliografía}

1. Soto-Estrada G, Moreno-Altamirano L, Pahua-Días D. Panorama epidemiológico de México, principales causas de morbilidad y mortalidad. Revista de la Facultad de Medicina de la UNAM. 2016;59:8-22.

2. American Thoracic Society. Guidelines for the management of adults with community-acquired pneumoniae. Am J Respir Crit Care Med. 2001; $163: 1730-54$

3. Mandell LA, Wunderink RG, Anzueto A, Bartlett JG, Campbell GD, Dean NC, et al. Infectious Diseases Society of Ameria/American Thoracic Society consensus guidelines on the management of community-acquired pneumonia in adults. Clin Infect Dis. 2007;44(Suppl 2):S27-S72.

4. Woodhead M, Blasi F, Ewig S, Huchon G, leven M, Ortqvist A, et al. Guidelines for the management of adult lower respiratory tract infections. Eur Respir J. 2005;26:1138-80.

5. Lim WS, Lewis S, Macfarlane T. Severity prediction rules in community acquired pneumonia: a validation study. Thorax. 2000;55:219-23.

6. Fine MJ, Auble TE, Yealy DM, Hanusa BH, Weissfeld LA, Singer DE et al. A prediction rule to indentify low-risk patients with community-acquired pneumonia. New Engl J Med. 1997;336:243-50.

7. Yealy DM, Auble TE, Stone RA, Lave JR, Meehan TP, Graff LG, et al. Effect of increasing the intensity of implementing pneumonia guidelines: a randomized, controlled trial. Ann Intern Med. 2005;143:881-94.

8. Atlas SJ, Benzer TI, Borowsky LH, Chang Y, Burnham DC, Metlay JP, et al. Safely increasing the proportion of patients with community-acquired pneumonia treated as outpatients: an interventional trial. Arch Intern Med. 1998;158:1350-6.

9. Báez Saldaña R, Gómez-Zamora C, López-Elizondo C, Molina-Corona H, Santillán Martínez A, Sánchez-Hernández J, et al. Neumonía adquirida en la comunidad. Revisión y actualización con una perspectiva orientada a la calidad de la atención médica. Neumol Cir Torax. 2013; 72(Supl 1):6-43.

10. Chow S, Shao J, Wang H. Sample size calculations in clinical research. 2nd ed. Chapman \& Hall/CRC Biostatistics Series; 2008. p. 106.

11. Mortensen EM, Coley CM, Singer DE, Marrie TJ, Obrosky S, Kapoor WN, et al. Causes of death for patients with community-acquired pneumonia. Arch Intern Med. 2002;162:1059-64.

12. Ramírez JA, Community-Acquired Pneumonia Organization Investigators. Worldwide perspective of the quality of care provided to hospitalized patients with community-acquired pneumonia: results from the CAPO international cohort study. Semin Respir Crit Care Med. 2005;26:543-52.

13. Labarere J, Stone RA, Obrosky S, Yealy DM, Meehan TP, Auble TE, et al. Factors associated with the hospitalization of low-risk patients with community-acquired pneumonia in a cluster-randomized trial. J Gen Intern Med. 2006;21:745-52. 